\title{
On the Molecular Limit of Coulomb Gases
}

\author{
Gian Michele Graf, Daniel Schenker
}

Theoretische Physik, ETH Hönggerberg, CH-8093 Zürich, Switzerland

Received: 1 November 1994

Abstract: We give a partially new analysis of the molecular nature of matter. A key feature is a property of the Coulomb potential as $\mathbb{R}^{3}$ is decomposed into simplices. A further application thereof is given in an appendix.

\section{Introduction}

A mixture of electrons and various kinds of nuclei consists of individual atoms and molecules, provided the temperature and the density are sufficiently low. Put differently, a gas of elementary particles is effectively described in this thermodynamic regime in terms of an ideal gas of composite particles. Different mathematical formulations and verifications of this fact have been given by Fefferman [4], by Conlon, Lieb and Yau [2], and by Macris and Martin [8]. See also [9, 10] for a discussion of the issues involved. With the present work we merely intend to offer a partially new proof. The reader familiar with the subject should proceed directly to Sect. 2.

The mixture shall consist of $S$ species of spinless particles with masses $\mathbf{M}=\left(M_{1}, \ldots, M_{S}\right)$ and charges $\mathbf{Q}=\left(Q_{1}, \ldots, Q_{S}\right) \in \mathbb{Z}^{S}$. We assume that all negatively charged particles are fermions, whereas the statistics of the other particles is irrelevant. Let $N_{k} \in \mathbb{N}$ be the number of particles of the $k^{\text {th }}$ species, and set $\mathbf{N}=\left(N_{1}, \ldots, N_{S}\right)$. The total number of particles is $N=\sum_{k=1}^{S} N_{k}$. The Hilbert space $\mathscr{H}_{\mathbf{N}, \Lambda}$ for $\mathbf{N}$ particles confined to an open set $\Lambda \subset \mathbb{R}^{3}$ is the subspace of $L^{2}(\Lambda)^{\otimes N}$ carrying the permutation symmetry appropriate to the given statistics. The Hamiltonian is

$$
H_{\mathbf{N}, \Lambda}=-\sum_{l=1}^{N} \frac{\Delta_{\Lambda, l}}{2 m_{i}}+\sum_{\substack{l, j=1 \\ i<1}}^{N} \frac{q_{l} q_{j}}{\left|x_{i}-x_{j}\right|}=: T_{\mathbf{N}, \Lambda}+V_{\mathbf{N}},
$$

where $\left(m_{l}, q_{l}\right)=\left(M_{k}, Q_{k}\right)$ if the $i^{\text {th }}$ particle belongs to the $k^{\text {th }}$ species. Here $\Delta_{\Lambda}$ is the Dirichlet Laplacian on $\Lambda$. If $\Lambda=\mathbb{R}^{3}$, the index $\Lambda$ is omitted. Variable particle numbers are accounted for by means of the Fock space and the Hamiltonian

$$
\mathscr{H}_{\Lambda}=\mathscr{F}\left(L^{2}(\Lambda)\right)=\bigoplus_{\mathbf{N}} \mathscr{H}_{\mathbf{N}, \Lambda}, \quad H_{\Lambda}=\bigoplus_{\mathbf{N}} H_{\mathbf{N}, \Lambda} .
$$


For bounded $\Lambda$, the grand canonical partition function and the (finite volume) pressure are given by

$$
\begin{aligned}
& \Xi(\beta, \boldsymbol{\mu}, \Lambda)=\operatorname{tr}_{\mathscr{H}} \mathbf{e}^{-\beta\left(H_{\Lambda}-\boldsymbol{\mu} \cdot \mathbf{N}\right)}=\sum_{\mathbf{N}} \operatorname{tr}_{\mathscr{N}} \mathbf{N}, \Lambda \\
& \mathbf{e}^{-\beta\left(H_{\mathbf{N}, \Lambda}-\boldsymbol{\mu} \cdot \mathbf{N}\right)}, \\
& p(\beta, \boldsymbol{\mu}, \Lambda)=(\beta|\Lambda|)^{-1} \log \Xi(\beta, \boldsymbol{\mu}, \Lambda),
\end{aligned}
$$

where $\beta>0$ is the inverse temperature and $\boldsymbol{\mu}=\left(\mu_{1}, \ldots, \mu_{S}\right) \in \mathbb{R}^{S}$ are the chemical potentials of the various species. The existence of the thermodynamic limit

$$
p(\beta, \boldsymbol{\mu})=\lim _{\Lambda \rightarrow \infty} p(\beta, \boldsymbol{\mu}, \Lambda)
$$

for suitable sequences $\{\Lambda\}$ (e.g. sequences of balls) has been proven by Lieb and Lebowitz [7]. They also proved that

$$
p(\beta, \boldsymbol{\mu})=p(\beta, \boldsymbol{\mu}+\lambda \mathbf{Q}) \quad(\lambda \in \mathbb{R})
$$

which expresses charge neutrality.

A basic version of the result [2,4] states that for suitable values of the chemical potentials $\mu_{0}$ and for low enough temperature $\beta^{-1}$ the pressure of the $S$ species is to good accuracy that of a classical free gas of specific "molecules." In this picture, molecules are non-interacting particles with no internal degrees of freedom. The types of molecules which actually occur are determined by the neutral ground states of $H-\boldsymbol{\mu}_{0} \cdot \mathbf{N}$, as we shall explain shortly. Let $E_{\mathbf{N}}$ and $E(\boldsymbol{\mu})$ be the ground state energies of $H_{\mathbf{N}}$, resp. of $H-\boldsymbol{\mu} \cdot \mathbf{N}$ except for the vacuum, i.e., let

$$
\begin{aligned}
E_{\mathbf{N}} & =\inf \left\{\left(\Psi, H_{\mathbf{N}} \Psi\right) \mid \Psi \in \mathscr{H}_{\mathbf{N}},\|\Psi\|=1\right\}, \\
E(\boldsymbol{\mu}) & =\inf _{\mathbf{N} \neq \mathbf{0}}\left(E_{\mathbf{N}}-\boldsymbol{\mu} \cdot \mathbf{N}\right) .
\end{aligned}
$$

Our assumption (A) on the chemical potentials $\boldsymbol{\mu}_{0}$ embodies the symmetry (1.4): There is $\lambda_{0} \in \mathbb{R}$ such that

$$
\boldsymbol{\mu}_{0}^{\prime}=\boldsymbol{\mu}_{0}+\lambda_{0} \mathbf{Q}
$$

enjoys the following two properties.

1) For some $\sigma>0$ and all $\mathbf{N}$,

$$
H_{\mathbf{N}}-\boldsymbol{\mu}_{0}^{\prime} \cdot \mathbf{N} \geqq \sigma N .
$$

A first consequence is that $E\left(\boldsymbol{\mu}_{0}^{\prime}\right)>0$ and that the set of "ground states",

$$
\mathscr{G}=\left\{\mathbf{N} \neq \mathbf{0} \mid E_{\mathbf{N}}-\boldsymbol{\mu}_{0}^{\prime} \cdot \mathbf{N}=E\left(\boldsymbol{\mu}_{0}^{\prime}\right)\right\},
$$

is non-empty and finite.

2) Either $\mathbf{Q} \cdot \mathbf{N}=0$ for all $\mathbf{N} \in \mathscr{G}$ (neutral case) or there are $\mathbf{N}_{+}, \mathbf{N}_{-} \in \mathscr{G}$ with $\pm \mathbf{Q} \cdot \mathbf{N}_{ \pm}>0$ (charged case).

The configuration space for $\mathbf{N}$ particles is

$$
X_{\mathbf{N}}=\left\{x=\left(x_{1}, \ldots, x_{N}\right) \mid x_{i} \in \mathbb{R}^{3}, i=1, \ldots, N\right\},
$$

that for their center of mass is $X_{\mathbf{N}}^{C}=\left\{x \in X_{\mathbf{N}} \mid x_{i}=x_{j}, i, j=1, \ldots, N\right\}$, and that for their relative position is $X_{\mathbf{N}}^{R}=\left\{x \in X_{\mathbf{N}} \mid \sum_{i=1}^{N} m_{l} x_{i}=0\right\}$. We have $X=X^{C} \oplus X^{R}$ as 
an orthogonal sum with respect to the inner product $x \cdot y=\sum_{i=1}^{N} m_{l} x_{l} y_{i}$. Explicitly, $x=x^{C}+x^{R}$ with

$$
x_{l}^{C}=x_{0}, \quad x_{i}^{R}=x_{l}-x_{0},
$$

where $x_{0}=(\mathbf{M} \cdot \mathbf{N})^{-1} \sum_{l=1}^{N} m_{i} x_{i}$ is the center of mass. Correspondingly, $\mathscr{H}_{\mathbf{N}}$ has a factorization $\mathscr{H}_{\mathbf{N}}=\mathscr{H}_{\mathbf{N}}^{C} \otimes \mathscr{H}_{\mathbf{N}}^{R}$ and $H_{\mathbf{N}}$ a decomposition $H_{\mathbf{N}}=T_{\mathbf{N}}^{C} \otimes 1+1 \otimes H_{\mathbf{N}}^{R}$. We remark that the kinetic energy $T_{\mathbf{N}}^{C}$ of the center of mass is unitarily equivalent to $-(2 \mathbf{M} \cdot \mathbf{N})^{-1} \Delta$ on $\mathrm{L}^{2}\left(\mathbb{R}^{3}, d x_{0}\right)$ and that $(1.5)$ applies to $H_{\mathrm{N}}^{R}$ as well. The HVZTheorem [3] implies

$$
\inf \sigma_{\mathrm{ess}}\left(H_{\mathbf{N}}^{R}-\boldsymbol{\mu}_{\mathbf{0}}^{\prime} \cdot \mathbf{N}\right) \geqq 2 E\left(\boldsymbol{\mu}_{0}^{\prime}\right) .
$$

It follows from (A1) that there is $0<g<E\left(\boldsymbol{\mu}_{0}^{\prime}\right)$ with

$$
\sigma_{\mathrm{d} 1 \mathrm{sc}}\left(H_{\mathbf{N}}^{R}-\boldsymbol{\mu}_{0}^{\prime} \cdot \mathbf{N}\right) \cap\left(-\infty, E\left(\boldsymbol{\mu}_{0}^{\prime}\right)+g\right) \subset\left\{E\left(\boldsymbol{\mu}_{0}^{\prime}\right)\right\}
$$

for all $\mathbf{N} \neq \mathbf{0}$. Note that $E\left(\boldsymbol{\mu}_{0}^{\prime}\right)$ is an eigenvalue of $H_{\mathbf{N}}^{R}-\boldsymbol{\mu}_{0}^{\prime} \cdot \mathbf{N}$ iff $\mathbf{N} \in \mathscr{G}$.

Proviso. We shall henceforth count $N \in \mathscr{G}$ repeatedly, according to the multiplicity of this eigenvalue.

The pressure of an ideal classical gas of molecules of composition $\mathbf{N}$, internal energy $E_{\mathbf{N}}$ and chemical potential $\mu$ is

$$
p_{\mathbf{N}}(\beta, \mu)=\frac{1}{\beta}\left(\frac{\mathbf{M} \cdot \mathbf{N}}{2 \pi \beta}\right)^{3 / 2} \mathbf{e}^{-\beta\left(E_{\mathbf{N}}-\mu\right)}
$$

Consider an ideal mixture of such gases with compositions $\mathbf{N} \in \mathscr{G}$. This notation is defined in thermodynamics by the additivity of partial pressures:

$$
p_{\mathscr{G}}(\beta, \boldsymbol{\mu})=\sum_{\mathbf{N} \in \mathscr{G}} p_{\mathbf{N}}(\beta, \boldsymbol{\mu} \cdot \mathbf{N}) .
$$

The chemical potentials on the r.h.s. correspond to chemical equilibrium among the molecules $\mathbf{N} \in \mathscr{G}$. The pressure $p_{\mathscr{G}}$ may not satisfy (1.4), i.e., it may be related to a non-neutral ensemble. This can happen because the molecules, although possibly charged, do not interact in this picture. One enforces (1.4) by setting

$$
p_{\mathscr{G}}^{0}(\beta, \boldsymbol{\mu})=\inf _{i \in \mathbb{R}} p_{\mathscr{g}}(\beta, \boldsymbol{\mu}+\lambda \mathbf{Q}) .
$$

Consider (A2). In the neutral case any $\lambda \in \mathbb{R}$ minimizes (1.9). In the charged case, $p_{\mathscr{G}}\left(\beta, \boldsymbol{\mu}+\lambda^{\prime} \mathbf{Q}\right)$ is a strictly convex function of $\lambda^{\prime}$ which diverges as $\left|\lambda^{\prime}\right| \rightarrow \infty$. Thus (1.9) has a unique minimizer $\lambda$. It tends to $\lambda_{0}$ as $(\beta, \boldsymbol{\mu}) \rightarrow\left(+\infty, \boldsymbol{\mu}_{0}\right)$. To see this, note that in this limit $\beta^{-1} \log p_{\mathscr{g}}\left(\beta, \boldsymbol{\mu}+\lambda^{\prime} \mathbf{Q}\right) \rightarrow-E\left(\boldsymbol{\mu}_{0}^{\prime}\right)+\max _{\mathbf{N} \in \mathscr{G}}\left(\lambda^{\prime}-\lambda_{0}\right) \mathbf{Q} \cdot \mathbf{N}$ uniformly in $\lambda^{\prime}$.

Theorem 1. Suppose assumption (A) holds. Then

$$
p(\beta, \boldsymbol{\mu})=p_{\mathscr{g}}^{0}(\beta, \boldsymbol{\mu})\left(1+O\left(\mathbf{e}^{-\varepsilon \beta}\right)\right)
$$

for some $\varepsilon>0$ in the limit $(\beta, \mu) \rightarrow\left(+\infty, \mu_{0}\right)$. 


\section{Discussion of the Proof}

In this work we shall only prove the upper bound

$$
p(\beta, \mu) \leqq p_{\mathscr{G}}^{0}(\beta, \mu)\left(1+O\left(\mathbf{e}^{-\varepsilon \beta}\right)\right) .
$$

(The opposite bound [2,4] relies on the variational principle). In [4] the bound (2.1), except for a different error term, is proven using an almost complete covering of $\mathbb{R}^{3}$ by means of disjoint balls. Essentially, the original Hamiltonian (1.1) is bounded below by one in which particles are confined to balls and the interaction between balls is dropped. In [2] a decomposition of $\mathbb{R}^{3}$ into cubes is used instead. We suggest to decompose $\mathbb{R}^{3}$ into simplices. An open simplex $\Delta \subset \mathbb{R}^{3}$, i.e. a tetrahedron, is a set

$$
\Delta=\left\{x \in \mathbb{R}^{3} \mid a_{l} x<c_{l}, i=1, \ldots, 4\right\}
$$

with $a_{l} \in \mathbb{R}^{3}, c_{l} \in \mathbb{R}$ and

$$
\begin{gathered}
\sum_{i=1}^{4} a_{i}=0, \\
\left|\operatorname{det}\left(a_{l}, a_{J}, a_{k}\right)\right|=\frac{1}{6} .
\end{gathered}
$$

Here $i, j, k$ is some (and, by (2.2), any) triple of distinct integers in $\{1, \ldots, 4\}$. The value $1 / 6$ is a convenient normalization. Elementary considerations show that the volume of $\Delta$ is

$$
|\Delta|=\left(\sum_{i=1}^{4} c_{i}\right)_{+}^{3},
$$

where $x_{+}=\max (x, 0)$. The reason for choosing simplices is contained in the following two lemmas.

The spherical average of a function $f: \mathbb{R}^{3} \rightarrow \mathbb{R}$ is the function $\bar{f}:[0,+\infty) \rightarrow$ $\mathbb{R}$ given by

$$
\bar{f}(r)=\int_{S^{2}} d \omega f(r \omega),
$$

where $d \omega$ is the normalized surface measure on the unit sphere $S^{2}=\left\{\omega \in \mathbb{R}^{3}|| \omega \mid=\right.$ 1\}. Alternatively,

$$
\bar{f}(|x|)=\int_{\mathrm{SO}(3)} d \mu(R) f\left(R^{-1} x\right),
$$

where $d \mu(R)$ is the Haar measure on $\mathrm{SO}(3)$.

Lemma 2. Let $\Delta$ be a simplex with characteristic function $\chi$. Set $\chi_{-}(x)=\chi(-x)$ and let $h(r)$ be the spherical average of $\chi * \chi_{-}$. Then $h \in C_{0}^{2}[0,+\infty), h(0)=|\Delta|$ and $h^{\prime \prime}(r)$ is non-increasing in $r$.

Lemma 3. Let $h \in C^{2}[0,+\infty)$ with $\lim _{r \rightarrow+\infty} h(r)=0$ and let $h^{\prime \prime}(r)$ be nonincreasing. Then

$$
w(x)=\frac{h(0)-h(|x|)}{|x|}, \quad\left(x \in \mathbb{R}^{3}\right)
$$

has positive Fourier transform: $\widehat{w}(p) \geqq 0$.

Here $h(0)|x|^{-1}$ is the Coulomb potential and, in a sense to be made precise later, $h(|x|)|x|^{-1}$ represents the same interaction restricted to particles belonging to 
the same simplex in a decomposition of $\mathbb{R}^{3}$. The following consequence of the positivity of the Fourier transform is well-known: Set

$$
V[w]=\sum_{\substack{l, j=1 \\ i<1}}^{N} q_{l} q_{j} w\left(x_{i}-x_{J}\right) .
$$

It follows from

$$
(2 \pi)^{3} \sum_{l, j=1}^{N} q_{l} q_{j} w\left(x_{l}-x_{j}\right)=\int d p \widehat{w}(p) \sum_{l, j=1}^{N} q_{l} q_{j} \mathbf{e}^{1 p\left(x_{l}-x_{l}\right)}=\int d p \widehat{w}(p)\left|\sum_{i=1}^{N} q_{i} \mathbf{e}^{\mathrm{i} p x_{l}}\right|^{2},
$$

that if $\widehat{w}(p) \geqq 0$ then

$$
V[w] \geqq-\frac{1}{2} w(0) \sum_{i=1}^{N} q_{i}^{2} .
$$

Due to the use of simplices, we shall not need the continuity of the stability of matter constant [2]. That continuity refers to $\sigma$ in (1.5) as a function of $v \downarrow 0$ when the Coulomb potential is replaced by a Yukawa potential

$$
Y_{v}(x)=\mathbf{e}^{-v|x|} /|x| \text {. }
$$

However we shall use, as in [2], that stability of matter with some constant holds for Yukawa potentials [1]. The rest of the proof of (2.1) is patterned after [2]. The plan is to find a simplex which is (i) so small that most likely it does not contain anything, but if it does, then most likely a molecule $\mathbf{N} \in \mathscr{G}$; (ii) so large that the energy essentially goes down when breaking $\mathbb{R}^{3}$ into such simplices. The outcome is given by the next two lemmas.

Lemma 4. Assume (A1). Let $\Delta$ be a simplex. Then there is $\varepsilon>0$ such that

$$
p\left(\beta, \boldsymbol{\mu}^{\prime}, \mathbf{e}^{\gamma / \beta} \Delta\right) \leqq p_{\mathscr{g}}\left(\beta, \boldsymbol{\mu}^{\prime}\right)\left(1+O\left(\mathbf{e}^{-\varepsilon \beta}\right)\right), \quad\left(\left(\beta, \boldsymbol{\mu}^{\prime}\right) \rightarrow\left(+\infty, \boldsymbol{\mu}_{0}^{\prime}\right)\right)
$$

for all small enough $\gamma>0$.

Lemma 5. There is a simplex $\Delta^{+}$such that

$$
p(\beta, \boldsymbol{\mu}) \leqq p\left(\beta, \boldsymbol{\mu}+O\left(l^{-1}\right), l \Delta^{+}\right)\left(1+O\left(l^{-1}\right)\right), \quad(l \rightarrow+\infty)
$$

uniformly in $\beta>0$ and $\boldsymbol{\mu} \in \mathbb{R}^{S}$.

Proof of (2.1). Let $\boldsymbol{\mu}^{\prime}=\boldsymbol{\mu}+\lambda \mathbf{Q}$, where $\lambda$ is the minimizer of (1.9) in the charged case and $\lambda=\lambda_{0}$ in the neutral one. Thus $\boldsymbol{\mu}^{\prime} \rightarrow \boldsymbol{\mu}_{0}^{\prime}$ in the limit considered. Using successively $(1.4),(2.9,8)$ and $(1.8)$ we obtain

$p(\beta, \boldsymbol{\mu})=p\left(\beta, \boldsymbol{\mu}^{\prime}\right) \leqq p_{\mathscr{G}}\left(\beta, \boldsymbol{\mu}^{\prime}+O\left(\mathbf{e}^{-\varepsilon \beta}\right)\right)\left(1+O\left(\mathbf{e}^{-\varepsilon \beta}\right)\right)=p_{\mathscr{G}}\left(\beta, \boldsymbol{\mu}^{\prime}\right)\left(1+O\left(\mathbf{e}^{-\varepsilon \beta}\right)\right)$, at the expense of possibly making $\varepsilon>0$ smaller.

\section{The Pressure in a Simplex}

We are going to derive (2.8). A similar bound for cubes has been obtained in [2]. The proof given there applies here too, because it does not significantly depend on the shape of the domain. We include it here for the convenience of the reader.

Proof of Lemma 4. We may assume $-\Delta \subset 3 \Delta$ upon translation of $\Delta$. In fact in this way we can make the $c_{i}(i=1, \ldots, 4)$ equal. Then, by (2.2), $x \in \Delta$ implies 
$a_{l}(-x)=\sum_{j \neq l} a_{j} x<\sum_{j \neq i} c_{j}=3 c_{i}$, i.e., $-x \in 3 \Delta$. For $\mathbf{N} \neq \mathbf{0}$ and $l>0$ let $X_{\mathrm{N}, l}^{\#}=$ $\left\{x \in X_{\mathrm{N}}^{\#} \mid x_{i} \in l \Delta, i=1, \ldots, N\right\}$, where \# stands for $C, R$ or is omitted. It then follows from (1.6) that

$$
X_{\mathbf{N}, l} \subset X_{\mathbf{N}, l}^{C}+X_{\mathbf{N}, 4 l}^{R} \subset X_{\mathbf{N}, 5 l}
$$

and in particular $H_{\mathbf{N}, l} \geqq T_{\mathbf{N}, l}^{C}+H_{\mathbf{N}, 4 l}^{R}$ in the sense of non-densely defined quadratic forms. Hence,

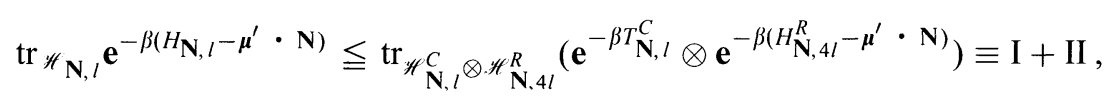

the splitting being as follows: Let $g>0$ be the gap in (1.7) and let $\mathscr{P}_{\mathrm{N}}$ be the subspace of $\mathscr{H}_{\mathrm{N}, 4 l}^{R}$ corresponding to the spectrum of $H_{\mathbf{N}, 4 l}^{R}-\boldsymbol{\mu}_{0}^{\prime} \cdot \mathbf{N}$ below $E\left(\boldsymbol{\mu}_{0}^{\prime}\right)+g$. Then I + II corresponds to $\mathscr{H}_{\mathbf{N}, 4 l}^{R}=\mathscr{P}_{\mathbf{N}} \oplus \mathscr{P} \frac{1}{\mathrm{~N}}$. To estimate I we use [5]

$$
\operatorname{tr}_{L^{2}(\Lambda)} \mathbf{e}^{\beta \Delta_{\Lambda}} \leqq(4 \pi \beta)^{-3 / 2}|\Lambda|
$$

for the first factor in (3.2) and $H_{\mathrm{N}, 4 l}^{R} \geqq E_{\mathrm{N}}$ for the second one, i.e.,

$$
\mathrm{I} \leqq\left(\frac{\mathbf{M} \cdot \mathbf{N}}{2 \pi \beta}\right)^{3 / 2}|l \Delta|\left(\operatorname{dim} \mathscr{P}_{\mathbf{N}}\right) \mathrm{e}^{-\beta\left(E_{\mathbf{N}}-\mu^{\prime} \cdot \mathbf{N}\right)} .
$$

We remark that $\operatorname{dim} \mathscr{P}_{\mathbf{N}}$ is bounded by the multiplicity of $\mathbf{N} \in \mathscr{G}$, since $H_{\mathbf{N}, 4 l}^{R} \geqq H_{\mathrm{N}}^{R}$. We will show below that on $\mathscr{P}_{\mathrm{N}}$,

$$
H_{\mathbf{N}, 4 l}^{R}-\boldsymbol{\mu}^{\prime} \cdot \mathbf{N} \geqq E\left(\boldsymbol{\mu}_{0}^{\prime}\right)+\frac{g}{2}+\delta\left(T_{\mathbf{N}, 4 l}^{R}+N\right)
$$

for some $0<\delta \leqq 1$, all $\mathbf{N} \neq \mathbf{0}$ and $\boldsymbol{\mu}^{\prime}$ close to $\boldsymbol{\mu}_{0}^{\prime}$. From (3.1) we have $T_{\mathbf{N}, l}^{C}+$ $T_{\mathrm{N}, 4 l}^{R} \geqq T_{\mathrm{N}, 5 l}$. Therefore,

$$
\mathrm{II} \leqq\left(\operatorname{tr}_{\mathscr{H}} \mathbf{N}, 5 l=\mathbf{e}^{-\beta \delta T} \mathbf{N}, 5 l\right) \mathbf{e}^{-\beta\left(\delta N+E\left(\mu_{0}^{\prime}\right)+(g / 2)\right)} \leqq \mathbf{e}^{-\beta\left((\delta N / 2)+E\left(\mu_{0}^{\prime}\right)+(g / 2)\right)}
$$

for large $\beta$ and $l=\mathbf{e}^{\gamma / \beta}$ with small enough $\gamma>0$. Here we estimated the first factor on the r.h.s. by (const $|l \Delta|)^{N}$ using again (3.3). Combining the above with the case $\mathbf{N}=\mathbf{0}$ we have

$$
\begin{aligned}
& \Xi\left(\beta, \boldsymbol{\mu}^{\prime}, \mathbf{e}^{\gamma \beta} \Delta\right) \leqq 1+\beta\left|\mathbf{e}^{\gamma \beta} \Delta\right| p_{\mathscr{G}}\left(\beta, \boldsymbol{\mu}^{\prime}\right)+\mathrm{const} \mathbf{e}^{-\beta\left(E\left(\boldsymbol{\mu}_{0}^{\prime}\right)+(g / 2)\right)}, \\
& p\left(\beta, \boldsymbol{\mu}^{\prime}, \mathbf{e}^{\gamma \beta} \Delta\right) \leqq p_{\mathscr{g}}\left(\beta, \boldsymbol{\mu}^{\prime}\right)+\mathrm{const} \mathbf{e}^{-\beta\left(E\left(\boldsymbol{\mu}_{0}^{\prime}\right)+(g / 2)\right)}=p_{\mathscr{G}}\left(\beta, \boldsymbol{\mu}^{\prime}\right)\left(1+O\left(\mathbf{e}^{-\beta g / 4}\right)\right),
\end{aligned}
$$

where we used $\log (1+x) \leqq x$ and $p_{\mathscr{G}}\left(\beta, \boldsymbol{\mu}^{\prime}\right) \geqq \mathbf{e}^{-\beta\left(E\left(\boldsymbol{\mu}_{0}^{\prime}\right)+(g / 4)\right)}$ for $\beta$ large and $\boldsymbol{\mu}^{\prime}$ close to $\mu_{0}^{\prime}$. To prove (3.4) we consider the inequalities on $\mathscr{P}_{\mathrm{N}}^{\perp}$,

$$
H_{\mathbf{N}, 4 l}^{R}-\boldsymbol{\mu}_{0}^{\prime} \cdot \mathbf{N} \geqq\left\{\begin{array}{l}
E\left(\boldsymbol{\mu}_{0}^{\prime}\right)+g \\
\sigma N \\
\frac{1}{2} T_{\mathbf{N}, 4 l}^{R}+2 E\left(\mu_{0}^{\prime}\right)+\boldsymbol{\mu}_{0}^{\prime} \cdot \mathbf{N},
\end{array}\right.
$$

where the latter follows from the unitary equivalence $(1 / 2) T_{\mathrm{N}}^{R}+V_{\mathrm{N}} \cong 2 H_{\mathrm{N}}^{R} \geqq 2 E_{\mathrm{N}}$. We add them with weights $1-2\left(\varepsilon+\varepsilon^{2}\right), 2 \varepsilon, 2 \varepsilon^{2}$ and obtain

$$
H_{\mathrm{N}, 4 l}^{R}-\boldsymbol{\mu}^{\prime} \cdot \mathbf{N} \geqq E\left(\boldsymbol{\mu}_{0}^{\prime}\right)+\frac{g}{2}+\varepsilon^{2} T_{\mathrm{N}, 4 l}^{R}+\varepsilon \sigma N,
$$


provided $\left(1-4\left(\varepsilon+\varepsilon^{2}\right)\right)(g / 2)-2\left(\varepsilon-\varepsilon^{2}\right) E\left(\boldsymbol{\mu}_{0}^{\prime}\right)+\varepsilon\left(\sigma N+2 \varepsilon \boldsymbol{\mu}_{0}^{\prime} \cdot \mathbf{N}\right)+\left(\boldsymbol{\mu}_{0}^{\prime}-\boldsymbol{\mu}^{\prime}\right) \cdot$ $\mathbf{N} \geqq 0$ for all $\mathbf{N} \neq \mathbf{0}$. This holds true for small $\varepsilon>0$ and $\boldsymbol{\mu}^{\prime}$ close to $\boldsymbol{\mu}_{0}^{\prime}$.

\section{Some Properties of Simplices}

Proof of Lemma 2. We begin with

$$
\chi * \chi_{-}(x)=\int d y \chi(x-y) \chi(-y)=\int d y \chi(y) \chi(y+x)=|\Delta \cap(\Delta-x)| .
$$

Note that $y \in \Delta \cap(\Delta-x)$ iff $a_{l} y<c_{i}$ and $a_{l} y<c_{l}-a_{i} x$ for $i=1, \ldots, 4$, i.e., iff

$$
a_{l} y<\min \left(c_{i}, c_{i}-a_{l} x\right)=c_{i}-\left(a_{i} x\right)_{+} \quad(i=1, \ldots, 4) .
$$

Hence $\Delta \cap(\Delta-x)$ is again a simplex. According to $(2.4)$ its volume is

$$
|\Delta \cap(\Delta-x)|=\left(\sum_{l=1}^{4} c_{l}-\left(a_{i} x\right)_{+}\right)_{+}^{3}=|\Delta|(1-k(\omega) r)_{+}^{3},
$$

where we set $x=r \omega\left(r \geqq 0, \omega \in S^{2}\right)$ and $k(\omega)=|\Delta|^{-1 / 3} \sum_{i=1}^{4}\left(a_{i} \omega\right)_{+}$. This last function is continuous on $S^{2}$ and has a positive minimum there. Indeed, if $k(\omega)=0$ for some $\omega \in S^{2}$ then $a_{i} \omega=0$ for $i=1, \ldots, 4$ because of (2.2). Together with (2.3), this would imply that the four vectors $a_{1}, a_{2}, a_{3}, \omega \in \mathbb{R}^{3}$ are linearly independent, which is impossible. As a result,

$$
h(r)=|\Delta| \int_{S^{2}} d \omega(1-k(\omega) r)_{+}^{3}
$$

has compact support. Its second derivative $h^{\prime \prime}(r)=6|\Delta| \int_{S^{2}} d \omega k(\omega)^{2}(1-k(\omega) r)_{+}$is continuous and non-increasing in $r$. (Moreover, $h$ has a continuous third derivative, but we do not need this fact.)

Proof of Lemma 3. We note that $h,-h^{\prime}, h^{\prime \prime} \geqq 0$. Passing to spherical coordinates we find for $p \neq 0$,

$$
\begin{aligned}
\widehat{w}(p) & =\lim _{\varepsilon \downarrow 0} \int d x \mathbf{e}^{-i p x} \mathbf{e}^{-\varepsilon|x|} w(x)=\frac{4 \pi}{|p|} \lim _{\varepsilon \downarrow 0} \int_{0}^{\infty} d r \sin (|p| r) \mathbf{e}^{-\varepsilon r}(h(0)-h(r)) \\
& =-\lim _{\varepsilon \downarrow 0} \frac{4 \pi}{\varepsilon^{2}+|p|^{2}} \int_{0}^{\infty} d r\left(\cos (|p| r)+\frac{\varepsilon}{|p|} \sin (|p| r)\right) \mathbf{e}^{-\varepsilon r} h^{\prime}(r) \\
& =-\frac{4 \pi}{|p|^{2}} \int_{0}^{\infty} d r \cos (|p| r) h^{\prime}(r)=\frac{4 \pi}{|p|^{3}} \int_{0}^{\infty} d r \sin (|p| r) h^{\prime \prime}(r) \\
& =\frac{4 \pi}{|p|^{4}} \sum_{k=0}^{\infty}(-1)^{k} \int_{0}^{\pi} d t \sin t h^{\prime \prime}\left(\frac{k \pi+t}{|p|}\right) \geqq 0 .
\end{aligned}
$$

The third and fifth equalities are obtained by partial integration. The series above is alternating because $h^{\prime \prime} \geqq 0$ is non-increasing. Hence the final inequality.

Lemma 2 fails if one replaces the characteristic function by a smeared out one. The following lemma is of remedy. Let $\phi_{0} \in C_{0}^{\infty}\left(\mathbb{R}^{3}\right)$ be spherically symmetric with $\phi_{0} \geqq 0, \int \phi_{0}=1$ and $\operatorname{supp} \phi_{0} \subset\{|x| \leqq 1\}$. Set $\phi(x)=\eta^{-3} \phi_{0}(x / \eta)$ with $\eta>0$. 
Lemma 6. Let $h_{0}$ satisfy the hypothesis of Lemma 3 and let $h:[0,+\infty) \rightarrow \mathbb{R}$ be the function which is well-defined by $h(|x|)=\left(h_{0} * \phi\right)(x)$, where $h_{0}=h_{0}(|x|)$. Then $h_{0}(0)+h_{0}^{\prime}(0) \eta \leqq h(0) \leqq h_{0}(0)$. For large $C>0$ and $0<\eta<C^{-1}$ the function

$$
\tilde{h}(r)=(1-C \eta) h(r)+C \eta \frac{h(0)}{1+(r / \eta)}
$$

satisfies the hypothesis of Lemma 3. Moreover, $\tilde{h}(0)=h(0)$ and $\tilde{h}^{\prime}(0)=-C h(0)$.

Proof. Note that $h$ and hence $\tilde{h}$ are smooth. All the statements except that $\tilde{h}^{\prime \prime}$ is non-increasing are immediate, and so will be that one once we prove $\tilde{h}^{\prime \prime \prime} \leqq 0$. Let $\omega \in S^{2}$. It follows from $h(r)=\int d y h_{0}(|r \omega-y|) \phi(y)$ that

$$
h^{\prime \prime}(r)=\int d y\left[h_{0}^{\prime \prime}(\rho)\left(\frac{r-\omega y}{|r \omega-y|}\right)^{2}+h_{0}^{\prime}(\rho)\left(\frac{y^{2}-(\omega y)^{2}}{|r \omega-y|^{3}}\right)\right] \phi(y),
$$

where $\rho=|r \omega-y|$. Let $r \geqq 3 \eta$ and note that $|y| \leqq \eta$ on the support of the integrand. Using that $h_{0}^{\prime \prime}(\rho)$ is non-increasing in $r$ we obtain

$$
h^{\prime \prime \prime}(r) \leqq 3 \int d y\left[\rho h_{0}^{\prime \prime}(\rho)-h_{0}^{\prime}(\rho)\right]\left(\frac{r-\omega y}{|r \omega-y|^{5}}\right)\left(y^{2}-(\omega y)^{2}\right) \phi(y) .
$$

The estimates $\rho h_{0}^{\prime \prime}(\rho)=h_{0}^{\prime \prime}(\rho) \int_{0}^{\rho} d x \leqq \int_{0}^{\rho} d x h_{0}^{\prime \prime}(x)=h_{0}^{\prime}(\rho)-h_{0}^{\prime}(0)$, as well as $0 \leqq r-\omega y \leqq \rho$ and $\rho \geqq(r+\eta) / 2$ then yield

$$
h^{\prime \prime \prime}(r) \leqq \operatorname{const}\left(-h_{0}^{\prime}(0)\right) \frac{\eta^{2}}{(r+\eta)^{4}}
$$

for $r \geqq 3 \eta$. We have $h^{\prime \prime \prime}(r)=\left.(\omega \nabla)^{3} h\right|_{\omega r}=\left.(\omega \nabla) h_{0} *(\omega \nabla)^{2} \phi\right|_{\omega r}$, where $h_{0}=$ $h_{0}(|x|)$ is Lipschitz with constant $-h_{0}^{\prime}(0)$. Hence $\left|h^{\prime \prime \prime}(r)\right| \leqq$ const $\left(-h_{0}^{\prime}(0)\right) \eta^{-2}$ for any $r \geqq 0$. Thus (4.2) also holds for $r<3 \eta$. Due to

$$
\frac{d^{3}}{d r^{3}} \frac{\eta}{1+(r / \eta)}=-6 \frac{\eta^{2}}{(r+\eta)^{4}}
$$

we have $\tilde{h}^{\prime \prime \prime} \leqq 0$ if $C h_{0}(0) \gg-h_{0}^{\prime}(0)$ and $\eta<C^{-1}$.

In order to control the potential energy corresponding to last term in (4.1) we will use a "stability of matter" result, namely that

$$
T+V\left[Y_{v}\right] \geqq- \text { const } N
$$

uniformly in $v \geqq 0$, where $Y_{v}$ is the Yukawa potential (2.7). This bound holds under the assumption on the statistics of particles made in the introduction. It follows immediately from the results in the Appendix of [1] by avoiding to set the number of spin degrees of freedom equal to $N$ (which corresponds to bosons). Let

$$
K(x)=\frac{1}{|x|(1+|x|)} .
$$

Since $(1+r)^{-1}=\int_{0}^{\infty} d v \mathbf{e}^{-v} \mathbf{e}^{-v r}$ we have $K(x)=\int_{0}^{\infty} d v \mathbf{e}^{-v} Y_{v}(x)$ and hence

$$
T+V[K]=\int_{0}^{\infty} d v \mathbf{e}^{-v}\left(T+V\left[Y_{v}\right]\right) \geqq-\operatorname{const} N .
$$




\section{The Localization Method}

We adopt the localization method of [2], except that we break up $\mathbb{R}^{3}$ into simplices instead of cubes. One way of doing this is to cut the unit cube $W=[0,1]^{3}$ with all planes passing through the centre and an edge or a face diagonal of $W$. This gives rise to congruent simplices $\Delta_{n} \subset W,(n=1, \ldots, 24)$. We omit giving a proof of this fact. The simplices $\Delta_{\alpha}=\Delta_{n}+z$ with $\alpha=(z, n) \in \mathbb{Z}^{3} \times\{1, \ldots, 24\}=: I$ yield a partition of $\mathbb{R}^{3}$ up to their boundaries. We then pick $\varphi_{0} \in C_{0}^{\infty}\left(\mathbb{R}^{3}\right)$ spherically symmetric with $\int \varphi_{0}^{2}=1$ and $\left\{\varphi_{0}(x) \neq 0\right\}=\{|x|<1 / 2\}$. Set $\varphi(x)=\eta^{-3 / 2} \varphi_{0}(x / \eta)$ and $j_{\alpha}=\left(\chi_{\alpha} * \varphi^{2}\right)^{1 / 2}$, where $\chi_{\alpha}$ is the characteristic function of $\Delta_{\alpha}$. It follows that

$$
\sum_{\alpha \in I} j_{\alpha}^{2}(x)=1, \quad\left(x \in \mathbb{R}^{3}\right) .
$$

Moreover, there are congruent simplices $\Delta_{\alpha}^{+}$with

$$
\begin{gathered}
\operatorname{supp} j_{\alpha} \subset \Delta_{\alpha}^{+}, \\
\left|\Delta_{\alpha}^{+}\right| \leqq\left|\Delta_{\alpha}\right|(1+O(\eta))
\end{gathered}
$$

as $\eta \downarrow 0$. Let us identify $n$ with $(0, n)$. As proven at the end of this section, $j_{n} \in C^{1}\left(\mathbb{R}^{3}\right)$ with $\left|\nabla j_{n}\right| \leqq$ const $\eta^{-1}$. This and $\left|\operatorname{supp} \nabla j_{n}\right|=O(\eta)$ imply

$$
\left\|\nabla j_{n}\right\|_{2}^{2} \leqq \text { const } \eta^{-1} \text {. }
$$

We remark that $j_{n}^{2} * j_{n-}^{2}=\left(\chi_{n} * \chi_{n-}\right) * \phi$, where $\phi=\eta^{-3} \phi_{0}(x / \eta)$ and $\phi_{0}=\varphi_{0}^{2} * \varphi_{0}^{2}$ has $\int \phi_{0}=1$. Let $h(r)$ be the spherical average of $\sum_{n=1}^{24} j_{n}^{2} * j_{n-}^{2}$ and $h_{0}(r)$ that of $\sum_{n=1}^{24} \chi_{n} * \chi_{n-}$. From (2.5) and the spherical symmetry of $\phi$ we obtain $h(|x|)=$ $\left(h_{0} * \phi\right)(x)$, where $h_{0}=h_{0}(|x|)$. Lemma 2 implies $h_{0}(0)=|W|=1$ and that $h_{0}, h$ satisfy the hypothesis of Lemma 6.

The following construction depends on $\eta, l>0$ although our notation will not reflect this for simplicity. For $\alpha \in I$, let $\mathscr{H}_{\alpha}=\mathscr{H}_{l \Delta_{\alpha}^{+}}$and $H_{\alpha}=H_{l \Delta_{\alpha}^{+}}$be the Fock space and the Hamiltonian for the simplex $l \Delta_{\alpha}^{+}$as given in (1.2). Let $\Lambda$ be a ball centered at the origin. Set

$$
I(\Lambda)=\left\{\alpha \in I \mid l R\left(\Delta_{\alpha}^{+}+y\right) \cap \Lambda \neq \varnothing \text { for some } y \in W, R \in \operatorname{SO}(3)\right\} .
$$

For later use, note that for fixed $\eta, l$,

$$
\lim _{|\Lambda| \rightarrow \infty}|\Lambda|^{-1} \sum_{\alpha \in I(\Lambda)}\left|l \Delta_{\alpha}\right|=1 .
$$

We define a Hilbert space and a Hamiltonian acting on it as direct integrals with constant fibers:

$$
\mathscr{H}_{I(\Lambda)}=\int_{W \times \mathrm{SO}(3)}^{\oplus} d y d \mu(R) \bigotimes_{\alpha \in I(\Lambda)} \mathscr{H}_{\alpha}, \quad H_{I(\Lambda)}=\int_{W \times \mathrm{SO}(3)}^{\oplus} d y d \mu(R) \sum_{\alpha \in I(\Lambda)} H_{\alpha} .
$$

In order to compare $\mathscr{H}_{I(\Lambda)}, H_{I(\Lambda)}$ with $\mathscr{H}_{\Lambda}, H_{\Lambda}$ given in (1.2) we define a map $J: \mathscr{H}_{\Lambda} \rightarrow \mathscr{H}_{I(\Lambda)}$. To this end, let $j_{y, R, \alpha}: L^{2}(\Lambda) \rightarrow L^{2}\left(l \Delta_{\alpha}^{+}\right)$be given by

$$
j_{y, R, \alpha} \psi(x)=j_{\alpha}(x / l) \psi(R(x+l y)) .
$$

Then let

$$
j_{y, R}: L^{2}(\Lambda) \rightarrow \bigoplus_{\alpha \in I \in(\Lambda)} L^{2}\left(l \Delta_{\alpha}^{+}\right), \quad j_{y, R}=\bigoplus_{\alpha \in I(\Lambda)} j_{y, R, \alpha}
$$


By passing to the Fock spaces over these Hilbert spaces we get the map

$$
\Gamma\left(j_{y, R}\right): \mathscr{F}\left(L^{2}(\Lambda)\right) \rightarrow \mathscr{F}\left(\bigoplus_{\alpha \in I(\Lambda)} L^{2}\left(l \Delta_{\alpha}^{+}\right)\right),
$$

which acts as the $N$-fold tensor product of $j_{y, R}$ on $N$-particle states. Note that $\mathscr{F}\left(L^{2}(\Lambda)\right)=\mathscr{H}_{\Lambda}$ and $\mathscr{F}\left(\bigoplus_{\alpha \in I(\Lambda)} L^{2}\left(l \Delta_{\alpha}^{+}\right)\right)=\bigotimes_{\alpha \in I(\Lambda)} \mathscr{F}\left(L^{2}\left(l \Delta_{\alpha}^{+}\right)\right)=\bigotimes_{\alpha \in I(\Lambda)} \mathscr{H}_{\alpha}$. Finally, we define

$$
J: \mathscr{H}_{\Lambda} \rightarrow \mathscr{H}_{I(\Lambda)}, \quad J=\int_{W \times \mathrm{SO}(3)}^{\oplus} d y d \mu(R) \Gamma\left(j_{y, R}\right) .
$$

The map $j_{y, R, \alpha}^{*}: L^{2}\left(l \Delta_{\alpha}^{+}\right) \rightarrow L^{2}(\Lambda)$ is given by

$$
j_{y, R, \alpha}^{*} \psi(x)=j_{\alpha}\left(R^{-1}(x / l)-y\right) \psi\left(R^{-1} x-l y\right) .
$$

Hence $j_{y, R}^{*} j_{y, R}: L^{2}(\Lambda) \rightarrow L^{2}(\Lambda)$ acts as multiplication by $\sum_{\alpha \in l(\Lambda)} j_{\alpha}^{2}\left(R^{-1}(x / l)-y\right)$. This function of $x \in \Lambda$ equals 1 because of $(5.1,5)$. We conclude that $J^{*} J=1$, i.e., that $J$ is an isometry.

Lemma 7. Let $\eta=l^{-1}$. Then

$$
H_{\Lambda} \geqq \kappa J^{*} H_{l(\Lambda)} J-\text { const } l^{-1} N
$$

for large $l$, where $0<\kappa \leqq 1$ and $\kappa=1+O\left(l^{-1}\right)$ as $l \rightarrow+\infty$.

Proof. We claim that

$$
J^{*} H_{I(\Lambda)} J=T_{\Lambda}+V\left[h(|x| / l)|x|^{-1}\right]+c l^{-2} \sum_{i=1}^{N}\left(2 m_{l}\right)^{-1},
$$

where $c=\sum_{n=1}^{24}\left\|\nabla j_{n}\right\|_{2}^{2} \leqq$ const $\eta^{-1}$ by (5.4). Indeed, on 1-particle states

$$
\begin{aligned}
\int_{W \times \mathrm{SO}(3)} d y d \mu(R) j_{y, R}^{*}\left(\bigoplus_{\alpha \in I(\Lambda)}-\Delta_{\alpha}\right) j_{y, R} & =\int_{W \times \mathrm{SO}(3)} d y d \mu(R) \sum_{\alpha \in I(\Lambda)} \tilde{j}_{\alpha}\left(-\tilde{\Delta}_{\alpha}\right) \tilde{j}_{\alpha} \\
& =-\Delta_{\Lambda}+c l^{-2}
\end{aligned}
$$

where $\Delta_{\alpha}$ and $\tilde{\Delta}_{\alpha}$ are the Dirichlet Laplacians on $l \Delta_{\alpha}^{+}$and on $l R\left(\Delta_{\alpha}^{+}+y\right)$, and $\tilde{j}_{\alpha}=j_{\alpha}\left(R^{-1}(x / l)-y\right)$. The second equality follows from $-\Delta=\sum_{\alpha \in I}\left[\tilde{j}_{\alpha}(-\Delta) \tilde{j}_{\alpha}-\right.$ $\left.\left(\nabla \tilde{j}_{\alpha}\right)^{2}\right]$, from $(5.2,5)$ and from $\int_{W} d y \sum_{z \in \mathbb{Z}^{3}}\left(\nabla j_{(z, n)}\right)^{2}(x-y)=\left\|\nabla j_{n}\right\|_{2}^{2}$. Similarly, on 2-particle states $j_{y, R}^{(1) *} j_{y, R}^{(2) *}\left(\bigoplus_{\alpha \in I(\Lambda)}\left|x_{1}-x_{2}\right|^{-1}\right) j_{y, R}^{(1)} j_{y, R}^{(2)}=\sum_{\alpha \in I(\Lambda)} \tilde{j}_{\alpha}^{(1)} \tilde{j}_{\alpha}^{(2)} \mid x_{1}-$ $\left.x_{2}\right|^{-1} \tilde{j}_{\alpha}^{(1)} \tilde{j}_{\alpha}^{(2)}$ and

$$
\begin{aligned}
& \int_{W \times \operatorname{SO}(3)} d y d \mu(R) \sum_{\alpha \in I(\Lambda)} \tilde{j}_{\alpha}^{(1)} \tilde{j}_{\alpha}^{(2)}\left|x_{1}-x_{2}\right|^{-1} \hat{j}_{\alpha}^{(1)} \tilde{j}_{\alpha}^{(2)} \\
& \quad=\left|x_{1}-x_{2}\right|^{-1} \int_{\operatorname{SO}(3)} d \mu(R) \sum_{n=1}^{24} j_{n}^{2} * j_{n-}^{2}\left(R^{-1}\left(x_{1}-x_{2}\right) / l\right) \\
& =\left|x_{1}-x_{2}\right|^{-1} h\left(\left|x_{1}-x_{2}\right| / l\right)
\end{aligned}
$$


where the superscripts $(i)$ refer to particles $i=1,2$. This relies on

$$
\int_{W} d y \sum_{z \in \mathbb{Z}^{3}} j_{(z, n)}^{2}\left(x_{1}-y\right) j_{(z, n)}^{2}\left(x_{2}-y\right)=j_{n}^{2} * j_{n-}^{2}\left(x_{1}-x_{2}\right) .
$$

This proves (5.8). Lemma 6,3 and (2.6) imply

$$
V\left[|x|^{-1}\right] \geqq \kappa V\left[h(|x|)|x|^{-1}\right]+C \eta V\left[(1+|x| / \eta)^{-1}|x|^{-1}\right]-\frac{C}{2} \sum_{l=1}^{N} q_{l}^{2},
$$

where $\kappa=h(0)^{-1}(1-C \eta)=1+O(\eta)$. We then replace $x$ by $x / l$, divide by $l$ and add the kinetic energy. We get

$$
H_{\Lambda} \geqq \kappa\left(T_{\Lambda}+V\left[h(|x| / l)|x|^{-1}\right]\right)+(1-\kappa) T_{\Lambda}+C \eta V[K]-\text { const } l^{-1} N,
$$

with $K$ given by (4.3). If $C$ is large enough, then $\kappa \leqq 1-C \eta / 2$ for small $\eta$. It thus follows from (4.4) that $(1-\kappa) T_{\Lambda}+C \eta V[K] \geqq(C \eta / 2)\left(T_{\Lambda}+2 V[K]\right) \geqq-$ const $\eta N$ because dropping the factor 2 amounts to a change of the charges $\mathbf{Q}$. The proof is completed by collecting estimates.

Proof of Lemma 5. Let $\tilde{\mu}_{i}=\mu_{i}+$ const $l^{-1}$, where the constant is the one in (5.7). Then

$$
\begin{aligned}
\Xi(\beta, \boldsymbol{\mu}, \Lambda) & \leqq \operatorname{tr}_{\mathscr{H}_{A}} \mathbf{e}^{-\beta J^{*}\left(\kappa H_{I(\Lambda)}-\tilde{\boldsymbol{\mu}} \cdot \mathbf{N}\right) J} \leqq \operatorname{tr}_{\mathscr{H}_{A}} J^{*} \mathbf{e}^{-\beta\left(\kappa H_{I(\Lambda)}-\tilde{\boldsymbol{\mu}} \cdot \mathbf{N}\right)} J \\
& \leqq \operatorname{tr}_{\mathscr{H}_{I(\Lambda)}} \mathbf{e}^{-\beta\left(\kappa H_{I(\Lambda)}-\tilde{\boldsymbol{\mu}} \cdot \mathbf{N}\right)}=\int_{W \times \operatorname{SO}(3)} d y d \mu(R) \prod_{\alpha \in I(\Lambda)} \operatorname{tr}_{\mathscr{H} \alpha} \mathbf{e}^{-\beta\left(\kappa H_{\alpha}-\tilde{\boldsymbol{\mu}} \cdot \mathbf{N}\right)},
\end{aligned}
$$

where the second bound is Peierls inequality [7]. Since $0<\kappa \leqq 1$ we have $\kappa H_{\alpha} \geqq$

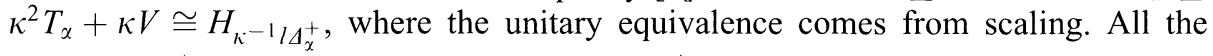
simplices $\Delta_{\alpha}^{+}$are congruent to a single one $\Delta^{+}$. We thus get

$$
p(\beta, \boldsymbol{\mu}, \Lambda) \leqq|\Lambda|^{-1}\left(\sum_{\alpha \in I(\Lambda)}\left|\kappa^{-1} l \Delta_{\alpha}^{+}\right|\right) p\left(\beta, \tilde{\boldsymbol{\mu}}, \kappa^{-1} l \Delta^{+}\right),
$$

and, in the limit $|\Lambda| \rightarrow \infty$,

$$
p(\beta, \boldsymbol{\mu}) \leqq|\Delta|^{-1}\left|\Delta^{+}\right| \kappa^{-3} p\left(\beta, \tilde{\boldsymbol{\mu}}, \kappa^{-1} l \Delta^{+}\right)=p\left(\beta, \boldsymbol{\mu}+O\left(l^{-1}\right), \kappa^{-1} l \Delta^{+}\right)\left(1+O\left(l^{-1}\right)\right),
$$

due to $(5.6,3)$. The function $l \mapsto \tilde{l}=\kappa^{-1} l$ is continuous with $\tilde{l} / l \rightarrow 1$ as $l \rightarrow+\infty$. Hence $O\left(l^{-1}\right)=O\left(\tilde{l}^{-1}\right)$.

Left to show is that our partition of unity is in $C^{1}\left(\mathbb{R}^{3}\right)$. Let $\chi \geqq 0$ any bounded (Borel) function, and $j=\left(\chi * \varphi^{2}\right)^{1 / 2}$, where $\varphi$ is as before. In particular, $\operatorname{supp} \varphi=$ $\{\varphi(x) \neq 0\}$ up to a Lebesgue null set. We claim that

$$
\nabla j= \begin{cases}(\chi * \varphi \nabla \varphi) j^{-1} & \text { if } j>0 \\ 0 & \text { if } j=0\end{cases}
$$

Only the second part requires proof. It means that if $\left(\chi * \varphi^{2}\right)(x)=0$ then $j(y)=o(|y-x|)$ as $y \rightarrow x$. Since $\chi(x-y)=0$ for a.e. $y \in \operatorname{supp} \varphi$ we have $\left(\chi * \partial_{x} \varphi^{2}\right)(x)=0$ for any multiindex $\alpha$. Hence, by the Taylor expansion with remainder, $j(y)^{2}=\left(\chi * \varphi^{2}\right)(y)=O\left(|y-x|^{n}\right)$ for any $n \geqq 0$. Continuity of $\nabla j$ is 
evident except for $\nabla j(y) \rightarrow 0$ as $y \rightarrow x$ with $j(x)=0$. By the Cauchy inequality, $|\chi * \varphi \nabla \varphi| \leqq\left(\chi * \varphi^{2}\right)^{1 / 2}\left(\chi *(\nabla \varphi)^{2}\right)^{1 / 2}$. This implies $(\nabla j)^{2} \leqq \chi *(\nabla \varphi)^{2}$. So, $\overline{\lim }_{y \rightarrow x}(\nabla j)^{2}(y) \leqq\left(\chi *(\nabla \varphi)^{2}\right)(x)=0$ and $|\nabla j|^{2} \leqq\|\chi\|_{\infty}\left\|(\nabla \varphi)^{2}\right\|_{1} \leqq$ const $\eta^{-2}$.

\section{Appendix. On the Continuity of the Free Energy}

We wish to illustrate another application of the decomposition into simplices. The thermodynamic limit for the free energy $f_{S}(\rho)$ of $S$ species (we drop $\beta$ ) exists for neutral densities, i.e., for $\rho$ in

$$
P_{S}=\left\{\boldsymbol{\rho}=\left(\rho_{1}, \ldots, \rho_{S}\right) \mid \boldsymbol{\rho} \cdot \mathbf{Q}=0, \rho_{i} \geqq 0, i=1, \ldots, S\right\} .
$$

This result is due to [7] to which we refer for the detailed statement. We intend to discuss the following fact $[7,6]$.

Proposition 8. $f_{S}(\boldsymbol{\rho})$ is continuous on $P_{S}$.

The set $P_{S}$ is a convex subset of $\left\{\boldsymbol{\rho} \in \mathbb{R}^{S} \mid \boldsymbol{\rho} \cdot \mathbf{Q}=0\right\}$. The convexity [7] of $f_{S}(\boldsymbol{\rho})$ implies that $f_{S}$ is upper semicontinuous on $P_{S}$ and continuous on its interior $\check{P}_{S}$. Upon relabelling species, $\boldsymbol{\rho}_{0} \in \partial P_{S}$ is of the form $\boldsymbol{\rho}_{0}=\left(\boldsymbol{\rho}_{0}^{\prime}, \mathbf{0}\right)$ with $\boldsymbol{\rho}_{0}^{\prime} \in \dot{P}_{S^{\prime}}$ and $0 \leqq S^{\prime}<S$. The lower semicontinuity of $f_{S}$ at $\rho_{\mathbf{0}} \in \partial P_{S}$,

$$
\varliminf_{\boldsymbol{\rho} \rightarrow \boldsymbol{\rho}_{0}} f_{S}(\boldsymbol{\rho}) \geqq f_{S^{\prime}}\left(\boldsymbol{\rho}_{0}^{\prime}\right),
$$

is proven in [6]. The proof rests on two main intermediate results. The first is the equivalence between the canonical and the grand canonical ensembles, namely

$$
p_{S}(\boldsymbol{\mu})=\sup _{\boldsymbol{\rho} \in P_{S}}\left[\boldsymbol{\rho} \cdot \boldsymbol{\mu}-f_{S}(\boldsymbol{\rho})\right] \text {, }
$$

which, incidentally, implies (1.4). The second is the statement "dual" to (A.1), i.e.,

$$
\varlimsup_{\boldsymbol{\mu} \rightarrow\left(\boldsymbol{\mu}_{0}^{\prime},-\infty\right)} p_{S}(\boldsymbol{\mu}) \leqq p_{S^{\prime}}\left(\boldsymbol{\mu}_{0}^{\prime}\right)
$$

the limit being $\mu_{i} \rightarrow \mu_{0 i}^{\prime}\left(i=1, \ldots, S^{\prime}\right)$ and $\mu_{i} \rightarrow-\infty\left(i=S^{\prime}+1, \ldots, S\right)$. The proof [6] of (A.3) is based on the decomposition [4] of $\mathbb{R}^{3}$ into balls. Our point is that it also follows from Lemma 5: Taking the above limit in (2.9) gives

$$
\varlimsup_{\boldsymbol{\mu} \rightarrow\left(\boldsymbol{\mu}_{0}^{\prime},-\infty\right)} p_{S}(\boldsymbol{\mu}) \leqq p_{S^{\prime}}\left(\boldsymbol{\mu}_{0}^{\prime}+O\left(l^{-1}\right), l \Delta^{+}\right)\left(1+O\left(l^{-1}\right)\right), \quad(l \rightarrow+\infty)
$$

because of the stated uniformity and the simple fact that $\varlimsup_{\boldsymbol{\mu} \rightarrow\left(\mu_{0}^{\prime},-\infty\right)} p_{S}\left(\mu, l \Delta^{+}\right) \leqq$ $p_{S^{\prime}}\left(\boldsymbol{\mu}_{0}^{\prime}, l \Delta^{+}\right)$for finite $l$. In the limit $l \rightarrow+\infty$ the r.h.s. in (A.4) tends to $p_{S^{\prime}}\left(\boldsymbol{\mu}_{0}^{\prime}\right)$ because, by convexity, the limit $(1.3)$ is locally uniform in $\mu$. Given $($ A.2, 3$)$ the proof [6] of (A.1) is immediate: We have $f_{S}(\boldsymbol{\rho}) \geqq \boldsymbol{\rho} \cdot \boldsymbol{\mu}-p_{S}(\boldsymbol{\mu})$ for all $\boldsymbol{\mu} \in \mathbb{R}^{S}$ and $f_{S^{\prime}}\left(\boldsymbol{\rho}_{0}^{\prime}\right)=\boldsymbol{\rho}_{0}^{\prime} \cdot \boldsymbol{\mu}_{0}^{\prime}-p_{S^{\prime}}\left(\boldsymbol{\mu}_{0}^{\prime}\right)$ for some $\boldsymbol{\mu}_{0}^{\prime} \in \mathbb{R}^{S^{\prime}}$ because $\boldsymbol{\rho}_{0}^{\prime} \in \check{P}_{S^{\prime}}$. We thus get

$$
\varliminf_{\boldsymbol{\rho} \rightarrow \boldsymbol{\rho}_{0}} f_{S}(\boldsymbol{\rho}) \geqq \boldsymbol{\rho}_{0} \cdot \boldsymbol{\mu}-p_{S}(\boldsymbol{\mu}),
$$

and, in the limit $\boldsymbol{\mu} \rightarrow\left(\boldsymbol{\mu}_{0}^{\prime},-\infty\right)$,

$$
\varliminf_{\boldsymbol{\rho} \rightarrow \boldsymbol{\rho}_{0}} f_{S}(\boldsymbol{\rho}) \geqq \boldsymbol{\rho}_{0}^{\prime} \cdot \boldsymbol{\mu}_{0}^{\prime}-p_{S^{\prime}}\left(\boldsymbol{\mu}_{0}^{\prime}\right)=f_{S^{\prime}}\left(\boldsymbol{\rho}_{0}^{\prime}\right) \text {. }
$$


Acknowledgements. G.M.G. thanks C. Fefferman and H.T. Yau for interesting discussions.

\section{References}

1. Conlon, J.G., Lieb, E.H., Yau, H.T.: The $N^{7 / 5}$ law for charged bosons. Commun. Math. Phys. 116, 417-448 (1988)

2. Conlon, J.G., Lieb, E.H., Yau, H.T.: The Coulomb gas at low temperature and low density. Commun. Math. Phys. 125, 153-180 (1989)

3. Cycon, H.L., Froese, R.G., Kirsch, W., Simon, B.: Schrödinger Operators. Berlin, Heidelberg, New York: Springer 1987

4. Fefferman, C.: The atomic and molecular nature of matter. Rev. Math. Iberoamericana 1, 1-44 (1985)

5. Fisher, M.E.: The free energy of a macroscopic system. Arch. Rat. Mech. Anal. 17, 377-410 (1964)

6. Hughes, W.: Thermodynamics for Coulomb systems: A problem at vanishing particle densities. J. Stat. Phys. 41, 975-1013 (1985)

7. Lieb, E.H., Lebowitz, J.L.: The constitution of matter: Existence of thermodynamics for systems composed of electrons and nuclei. Adv. in Math. 9, 316-398 (1972)

8. Macris, N., Martin, Ph.A.: Ionization equilibrium in the electron proton gas. J. Stat. Phys. 60, 619-637 (1990)

9. Martin, Ph.A.: Lecture on Fefferman's proof of the atomic and molecular nature of matter. Acta Physica Polonica P24, 751-770 (1993)

10. Peierls, R.: Surprises in Theoretical Physics. Princeton, NJ: Princeton University Press, 1979

Communicated by B. Simon 
